\title{
De Boston à Caacupé : l'échec international du projet One Laptop Per Child (OLPC)
}

\section{Damien Larrouqué}

\section{Q OpenEdition}

1 Journals

Édition électronique

URL : https://journals.openedition.org/ries/6022

DOI : $10.4000 /$ ries.6022

ISSN : 2261-4265

Éditeur

France Education international

\section{Édition imprimée}

Date de publication : 1 décembre 2017

Pagination : 15-18

ISBN : 978-2-85420-616-6

ISSN : $1254-4590$

Référence électronique

Damien Larrouqué, « De Boston à Caacupé : l'échec international du projet One Laptop Per Child (OLPC) », Revue internationale d'éducation de Sèvres [En ligne], 76 | décembre 2017, mis en ligne le 01 décembre 2019, consulté le 24 juin 2021. URL : http://journals.openedition.org/ries/6022 ; DOI : https://doi.org/10.4000/ries.6022

Ce document a été généré automatiquement le 24 juin 2021.

(c) Tous droits réservés 


\title{
De Boston à Caacupé : l'échec international du projet One Laptop Per Child (OLPC)
}

\author{
Damien Larrouqué
}

1 Imaginé par une équipe d'ingénieurs du Massachusetts Institute of Technology (MIT), le projet OLPC avait pour objectif de réduire la « brèche digitale » à l'échelle de la planète. Concrètement, il était question de lutter contre l'inégal accès aux NTIC, en convainquant les pouvoirs publics des pays du Sud d'investir dans des ordinateurs portables (laptops) très bon marché et spécifiquement dédiés à l'usage pédagogique (baptisés XO), puis de les distribuer gratuitement à leurs élèves. L'imparfait s'impose car, trop radical dans ses fondements, miné par la crise financière et peu probant en matière pédagogique, ce projet a finalement connu un destin moins glorieux que ce que laissait prédire l'enthousiasme international qu'il a soulevé à ses débuts.

\section{Les visions socio-pédagogiques controversées d'une fondation minée par la crise}

2 Synthétisé sous le slogan de "l'ordinateur à 100 dollars », le projet One Laptop Per Child a été dévoilé à la communauté internationale lors $\mathrm{du} 35^{\mathrm{e}}$ Forum économique mondial de Davos, en janvier 2005. Son instigateur, Nicholas Negroponte est considéré comme un génie de l'informatique. Ancien directeur du MIT Media Lab, le président de la fondation OLPC n'en est pas moins décrié pour sa confiance démesurée dans les outils numériques.

Cristallisé dans le projet OLPC, son » déterminisme technologique » (Garrido, 2009) va totalement à l'encontre des méthodes d'enseignement traditionnelles, au point de convertir le professeur en un simple appendice de l'outil informatique et d'appeler à sa disparition. Le XO a ainsi été pensé pour favoriser l'autonomie absolue de l'écolier: intuitive, sa prise en main ne nécessite pas l'intervention de l'éducateur. D'après ses 
contempteurs, le projet OLPC aurait confondu la fin de l'éducation (l'apprentissage) avec le moyen (la technologie numérique).

4 Toutefois, au-delà des ressentiments du corps enseignant, ce sont les déboires accumulés par sa fondation mère qui ont porté un coup fatal au déploiement international de cette initiative, laquelle a officiellement été abandonnée en mars 2014. De fait, le déclenchement de la crise économique au mois de septembre 2008 a entraîné le désengagement financier de nombreuses entreprises de l'industrie high-tech. À court de fonds, concurrencée par la mise sur le marché d'ordinateurs portables (laptops) commerciaux à bas coût, et incapable (pour des raisons d'économie d'échelle) de fournir aux gouvernements intéressés par son initiative un prix unitaire de 100 dollars USD par machine, la fondation de Boston a commencé à péricliter.

Malgré l'alourdissement de la facture pour les pouvoirs publics, OLPC a suscité un fort enthousiasme international. Au-delà de l'Afrique - où quelque $120000 \mathrm{XO}$ ont été distribués au Rwanda entre 2007 et 2009 -, c'est en Amérique latine, et notamment en Uruguay et au Pérou (presque 1,5 million de XO achetés à eux deux) que ce projet socioéducatif a été le plus largement diffusé. Ainsi, plus de $80 \%$ des XO produits à l'échelle internationale ont été répartis en Amérique latine (Cristia et al., 2012). Par la suite, le projet de Negroponte a inspiré des programmes similaires mais totalement indépendants par rapport à la fondation nord-américaine, tels que les plans Cainama Educativo au Venezuela ou Conectar Igualdad en Argentine.

\section{Les expériences latino-américaines passées au crible}

6 Du fait de son antériorité par rapport aux autres programmes du même type, d'une part, et des récompenses qu'il s'est vu octroyer par de nombreuses organisations internationales, d'autre part, le plan Ceibal uruguayen a fait l'objet de très nombreuses études d'impact. Compte tenu des limites bibliographiques imposées par cette rubrique, il nous est impossible de les citer toutes. Pour résumer, bien qu'elles rendent compte d'une très forte valorisation sociale de la politique publique et d'une plus grande motivation des élèves depuis l'arrivée des ordinateurs dans les classes, ces investigations se font également l'écho des principales critiques des utilisateurs: manque de formation selon les professeurs, fragilité des XO pour les enfants et défaut de contrôle informatique au regard de leurs parents. Très souvent invoquée, l'insuffisance de la formation des enseignants a conduit à un usage de l'ordinateur en classe faiblement assimilé et peu homogène, car variant en fonction de l'intérêt et des compétences du professeur en matière informatique. Au niveau pédagogique, des chercheurs de l'Université de la République (UdelaR) n'ont relevé aucun impact significatif sur l'apprentissage de la lecture et des mathématiques «ni au niveau général, ni en fonction du niveau socio-économique [des élèves]» (Machado et al., 2013). Au demeurant, toutes les investigations ont mis en évidence l'effet positif du plan Ceibal en termes d'inclusion sociale et territoriale.

7 Entre 2007 et 2011, avec quelque 900000 XO souscrits auprès de la fondation OLPC contre un peu moins de 400000 en Uruguay, le Pérou a été le plus grand client et partenaire de Negroponte au niveau mondial. C'est aussi la raison pour laquelle le pays a accueilli l'étude d'impact la plus ambitieuse. Financée par la Banque interaméricaine de développement (BID), cette investigation indépendante a été menée entre octobre et novembre 2010, soit une vingtaine de mois après le lancement du plan Una Computadora 
Por Niño (UCPN). Publiée deux ans plus tard, elle a rendu des conclusions assez embarrassantes pour la fondation OLPC comme pour le gouvernement d'Alan García. D'une part, les laptops éducatifs ne permettent pas d'améliorer l'apprentissage des matières fondamentales. D'autre part et au-delà du fait qu'il n'a pas été observé au Pérou une plus grande motivation des élèves et donc une baisse significative de la désertion scolaire, l'étude commanditée par la BID a laissé entendre que, loin de favoriser l'autostimulation intellectuelle des enfants, la confrontation avec le XO pouvait avoir des effets contre-productifs sur leur confiance en eux (Cristia et al., 2012).

Dès 2010, un couple de chercheurs californiens s'était montré moins bienveillant encore à l'égard du projet OLPC (Ames et Warshaueur, 2010). Sur la base de l'expérience-pilote lancée dans l'Alabama et des trois programmes d'alphabétisation numérique latino-américains les plus ambitieux (plan Ceibal, plan UCPN et projet de Caacupé au Paraguay), Ames et Warshaueur ont mis en lumière certains problèmes inhérents aux transpositions locales comme nationales d'OLPC : coûts financiers très importants, défaut de formation des enseignants, mauvaise qualité des XO et inadaptation aux contextes éducatifs singuliers (notamment multilingue ou pluriethnique). Pire, Laura Quispe (2015) a montré que les XO étaient aujourd'hui largement délaissées par le corps enseignant péruvien.

9 En Argentine, si le gouvernement de Cristina Kirchner a choisi de distribuer des ordinateurs plus performants que ceux proposés par la fondation du MIT, le programme Conectar Igualdad (CI) a néanmoins soulevé des réserves similaires, mais également suscité un enthousiasme équivalent à celui observé en Uruguay. En novembre 2011, le ministère de l'éducation a dévoilé les premiers résultats d'une investigation menée en partenariat avec onze universités publiques (Villanueva et al., 2011). Après un an de mise en œuvre du plan $\mathrm{CI}$, cette investigation a mis en exergue la très forte adhésion de la communauté enseignante au projet, son effet positif sur la motivation des élèves et ses bénéfices en termes d'intégration sociale. Par ailleurs, une chercheuse a souligné que la formation enseignante avait été l'un des points forts du plan CI : entre 2010 et 2013, presque 300000 professeurs de l'enseignement secondaire auraient ainsi été formés à l'outil informatique (Vacchieri, 2013). Cependant, il n'a pas été constaté une amélioration substantielle des résultats scolaires. Au mieux, l'effet pédagogique du plan $\mathrm{CI}$ a été jugé « naissant » ou « balbutiant » (ibid).

10 Le projet OLPC entendait révolutionner les politiques éducatives en introduisant l'innovation numérique dans les classes des pays dit «en développement ». Cependant, véhiculant une vision de l'enseignement déshumanisé et affaiblie par la crise financière, la fondation du MIT qui le défendait a rapidement périclité. Les évaluations réalisées à partir des expériences latino-américaines ont surtout démontré son inefficacité pédagogique. L'ordinateur ne favorise pas l'apprentissage. En revanche, ces programmes contribuent à la justice sociale. 


\section{BIBLIOGRAPHIE}

AMES M., WARSCHAUER M. (2010): « Can One Laptop per Child Save The World's Poor? », Journal of International Affairs, $n^{\circ} 64$, vol. 1, p. 33-51.

CRISTIA J. et al. (2012) : Tecnología y desarrollo en la niñez : evidencia del programa Una Laptop por Niño, Washington DC, BID.

GARRIDO F (2009) : « ¿ Otra vez el mismo error ? OLPC, determinismo tecnológico y educación », in R. Balaguer (dir.) : Uruguay : una computadora para cada Niño. Plan Ceibal. Los ojos del mundo en el primer modelo OLPC a escala nacional, Montevideo, Prentice Hall, p. 33-44.

LAURA QUISPE C. (2015) : «Maestros y computadoras portátiles en el Perú : ¿ Por qué no se usan las computadoras portátiles?», Apertura, vol. 7, n 1, p. 1-18.

MACHADO A. et al. (2013) : Profundizando en los efectos del Plan Ceibal, Montevideo, UdelaR.

VACCHIERI A (2013) : Las políticas TIC en los sistemas educativos de América latina : Caso Argentina, Buenos Aires, Unicef.

VILLANUEVA E. et al. (2011) : Nuevas voces, nuevos escenarios : estudios evaluativos sobre el programa Conectar Igualdad, Buenos Aires, Ministerio de Educación.

\section{INDEX}

Keywords : new technologies, computer assisted instruction, international cooperation project, digital

Palabras claves : nuevas tecnologías, enseñanza asistida por ordenador, proyecto de cooperación internacional, digital

Mots-clés : nouvelles technologies, apprentissage assisté par ordinateur, projet de cooperation internationale, numérique

Index géographique : États-Unis, Amérique latine, Uruguay, Argentine, Pérou

\section{AUTEUR}

\section{DAMIEN LARROUQUÉ}

Damien Larrouqué est attaché temporaire d'enseignement et de recherche (ATER) à l'Université Paris 2 Panthéon-Assas, docteur associé au Centre de recherches internationales (CERI-Sciences Po) et membre du bureau exécutif de l'Observatoire politique de l'Amérique latine et des Caraïbes (OPALC-Sciences Po). Ses recherches portent sur les transformations de l'État et de l'action publique en Amérique latine, ainsi que sur l'évolution des partis politiques, notamment en Argentine, au Paraguay et en Uruguay. Courriel: damien.larrouque@sciencespo.fr 